\title{
AN UPDATED AND ANNOTATED CHECKLIST OF BULGARIAN TERRESTRIAL GASTROPODS (MOLLUSCA: GASTROPODA)
}

\author{
ATANAS IRIKOV ${ }^{1}$, ZOLTÁN ERÖSS ${ }^{2}$ \\ ${ }^{1}$ Paisii Hilendarsky Plovdiv University, Department of Ecology and Nature Conservation, 24 Tzar Assen Str., \\ Plovdiv 4000, Bulgaria (e-mail: irikov@uni-plovdiv.bg) \\ ${ }^{2} 36$ Bem Str., Budapest 1151, Hungary (e-mail: erosspeter@yahoo.co.uk) \\ ABSTRACT: According to the current state of knowledge the terrestrial gastropod fauna of Bulgaria includes \\ 226 species and 33 subspecies (excluding nominate ones), making altogether 259 taxa. The following new \\ data are presented: updated nomenclature of 50 taxa, synonymisation of 17 taxa; taxa whose presence in Bul- \\ garia was discovered and those which were described as new to the science after the publication of the last \\ checklist (1998) are now included, six taxa are evaluated as introduced; the taxonomic status of 13 taxa is re- \\ garded as unclear. Fifty seven taxa are regarded as endemic to Bulgaria, and 33 as endemic to the Balkan Pen- \\ insula; 60 taxa are regarded as rare.
}

KEY WORDS: terrestrial gastropods, Bulgaria, checklist

\section{INTRODUCTION}

In the past mostly foreign and recently native specialists investigated the land gastropods in Bulgaria. The first and only comprehensive work on the Bulgarian terrestrial malacofauna was the monograph of DAMJANOV \& LIKHAREV (1975) - Fauna Bulgarica, Gastropoda terrestria. The authors (DAMJANOV \& LIKHAREV 1975) summarised all the then available data, except a few papers (URBAŃSKI 1969, HUDEC \& VAŠATKO 1973, NORDSIECK 1973, 1974). DAMJANOV \& LIKHAREV (1975) expressed an opinion that "in the near future it is difficult to expect finding any considerable number of new species in our country". After the publication of this monograph the malacofaunistic research in Bulgaria decreased in intensity. In 1975-1998 only three articles concerning Bulgarian mollusc fauna appeared: Claus (1977), JACKIEWICZ (1979) and KÖRNIG (1983). Later DEDOV (1998) presented the first checklist of Bulgarian terrestrial gastropods, updating the systematic status of some taxa. However, some papers were omitted when preparing this list: SAJÓ (1968), URBAŃSKI (1969),
PINTÉR L. (1972), RIEDEL (1975, 1980, 1998), NORDSIECK $(1970,1973,1974,1977)$, JACKIEWICZ (1979), RIEDEL \& MAASSEN (1993), GITTENBERGER \& BANK 1996 etc.; as a result some taxa were left out while the nomenclature of others was out-dated. In 1998-2008 the research on Bulgarian terrestrial malacofauna was carried out mostly by Bulgarian specialists. As a result, much information about taxonomy, distribution and ecology accumulated during the ten year period. Seven new taxa were described as new to the science (IRIKOV 2003, 2006, IRIKOV et al. 2004, DEDOV 2007); eight taxa were recorded as new to the fauna of Bulgaria (IRIKOV et al. 2004, IRIKOV 2006, 2008 in press, GEORGIEV in press), and one species was synonymised (IRIKOV 2001). The aim of this paper is to present a new, up-dated checklist, incorporating the new data accumulated since 1998. Notwithstanding the updating of the species list, recently many new forms (Macedonica, Agardhiella, Balea etc.) have been found in Bulgaria by various collectors; they are not included and are currently being described. 
The systematic arrangement and nomenclature follow DEDOV (1998). The following symbols are used in the list:

- species and subspecies with nomenclatural changes after publication of DEDOV's (1998) list: taxa transferred to another genus or with changed specific/subspecific status;

$\mathbf{S}$ taxa synonymised after publication of DEDOV's (1998) list;

I taxa introduced in Bulgaria;

- taxa omitted in DEDOV (1998);

! taxa recorded as new to Bulgarian fauna after publication of DEDOV's (1998) list;

!! taxa described as new to the science after publication of DEDOV's (1998) list;

\# taxa not valid, or not occurring in Bulgaria, or taxa whose occurrence in the country is uncertain;

? taxa with unclear systematic status;

$\approx$ taxa recorded from the Bulgarian Black Sea coast but not being a part of the native fauna (flood debris of the sea);

BGE - taxa endemic to Bulgaria;

BLE - taxa endemic to the Balkan Peninsula;

RARE - taxa with only one or few records.

\section{CHECKLIST}

Subclass Prosobranchia

Order Caenogastropoda

Superfamily Littorinoidea

Family Pomatiasidae

1. Pomatias rivulare (Eichwald, 1829)

2. Pomatias elegans (O. F. Müller, 1774)

Superfamily Cyclophoroidea

Family Aciculidae

3. Platyla polita (Hartmann, 1840)

4. Platyla similis (Reinhardt, 1880)

5. Platyla orthostoma (Jackiewicz, 1979) — RARE

Subclass Pulmonata

Order Bassommatophora

Superfamily Ellobioidea

Family Ellobiidae

6. Carychium minimum O. F. Müller, 1774

7. Carychium tridentatum (Risso, 1826)

8. Myosotella myosotis (Draparnaud, 1801)

Order Stylommatophora

Superfamily Pupilloidea

Family Pyramidulidae

9. Pyramidula pusilla (Vallot, 1801) • !

Pyramidula rupestris rupestris (Draparnaud, 1801) \# S

Pyramidula rupestris saxatilis Hartmann, 1840 \# S

The above two subspecies are listed in DEDOV (1998), but the specimens from the Rhodopes and the Pirin
Mts. examined by us turned out to be $P$. pusilla. Till finding specimens of $P$. rupestris we assume that only $P$. pusilla is present in Bulgaria.

Family Vertiginidae

I. Subfamily Vertigininae

10. Vertigo (Vertigo) pusilla O. F. Müller, 1774

11. Vertigo (Vertigo) substriata (Jeffreys, 1830) RARE

12. Vertigo (Vertigo) antivertigo (Draparnaud, 1801)

13. Vertigo (Vertigo) moulinsiana (Dupuy, 1849) RARE

14. Vertigo (Vertigo) pygmaea (Draparnaud, 1801)

15. Vertigo (Vertigo) alpestris (Alder, 1830)

16. Vertigo (Vertilla) angustior (Jeffreys, 1830)

II. Subfamily Truncatellininae

17. Truncatellina claustralis (Gredler, 1856)

18. Truncatellina cylindrica (Férussac, 1807)

19. Truncatellina costulata (Nilsson, 1822)

20. Truncatellina rothi (Reinhardt, 1916) ! RARE

This species, from the Western Rhodopes, is new to the Bulgarian fauna (IRIKOV 2008 in press).

Truncatellina callicratis (Scacchi, 1833) \#

In DEDOV (1998) Truncatellina callicratis (Scacchi, 1833) is listed as a synonym of Truncatellina strobeli (Gredler, 1853), but according to IRIKOV \& MOLLOV (2006) and IRIKOV (2008 in press) till now there is no evidence for the presence of this species in Bulgaria.

21. Columella edentula (Martens, 1830)

Family Pupillidae

Subfamily Pupillinae

22. Pupilla muscorum (Linnaeus, 1758)

23. Pupilla triplicata (Studer, 1820)

24. Pupilla sterri (Voith, 1838)

Pupilla bogdanovi Urbański, 1960 \# $\approx$

Pupilla valkanovi Urbański, 1960 \# $\approx$

The last two species: $P$. bogdanovi and $P$. valkanovi are included in DEDOV (1998) based on the material from the region of Bourgas, described by URBAŃSKI (1960). The species were described on the basis of a single shell each. After the original description there were no records of any of them either in Bulgaria or outside the country. It is very likely that the shells were brought by sea currents from other regions (not Bulgarian Black Sea coast). The origin and distribution of these species still remain unknown.

Family Lauridae

25. Lauria cylindracea (Da Costa, 1778)

26. Euxinolauria schweigeri (Götting, 1963) • !

This species is erroneously reported in DAMAJANOV \& LIKHAREV (1975) as Lauria (Leiostyla) anglica (Wood, 1828) and in DEDOV (1998) as Leiostyla anglica.

Family Argnidae

For information on Agardhiella in Bulgaria see IRIKOV \& GEORGIEV (2008 in press). The genus in still insufficiently studied and the existing material is too scanty 
for a comprehensive and reliable conchological and anatomical analysis. It is very likely that conchologically indistinguishable species may occur in Bulgaria, and more detailed research is needed.

27. Agardhiella truncatella (L. Pfeiffer, 1841) • RARE 28. Agardhiella macrodonta (Hesse, 1916) • BGE RARE In DEDOV (1998) recorded as the nominate subspecies Argna macrodonta macrodonta (Hesse, 1916). 29. Agardhiella rumelica (Hesse, 1916)

In DEDOV (1998) listed as Argna macrodonta rumelica (Hesse, 1916).

30. Agardhiella parreyssii (L. Pfeiffer, 1848) • BLE RARE

Family Orculidae

31. Orculella bulgarica (Hesse, 1915) RARE

In DEDOV (1998) listed as Orculella bulgarica bulgarica (Hesse, 1915).

32. Orcula zilchi Urbański, 1960

33. Sphyradium doliolum (Bruguière, 1792)

34. Pagodulina subdola brabeneci Hudec et Vašatko, 1971 BGE

Family Chondrinidae

35. Granaria frumentum (Draparnaud, 1801)

36. Chondrina avenacea (Bruguière, 1792)

37. Chondrina arcadica bulgarica (Nordsieck, 1970)

In DEDOV (1998) the species is listed as Chondrina

clienta (Westerlund, 1883), though according to

NORDSIECK (1970) the Bulgarian records actually pertain to Chondrina arcadica bulgarica.

Chondrina spelta (Beck, 1837) \#

Family Valloniidae

Subfamily Valloniinae

38. Vallonia costata (O. F. Müller, 1774)

39. Vallonia pulchella (O. F. Müller, 1774)

40. Vallonia enniensis (Gredler, 1856)

41. Vallonia excentrica Sterki, 1892

Family Strobilopsidae

I. Subfamily Acanthinulinae

42. Acanthinula aculeata (O. F. Müller, 1774)

II. Subfamily Speleodiscinae

43. Aspasita triaria triaria (Rossmässler, 1839) • RARE

Superfamily Cochlicopoidea

Family Cochlicopidae

44. Cochlicopa lubrica (O. F. Müller, 1774)

45. Cochlicopa lubricella (Porro, 1838)

46. Cochlicopa nitens (Gallenstein, 1852) RARE

Superfamily Buliminoidea

Family Enidae

I. Subfamily Eninae

47. Merdigera obscura (O. F. Müller, 1774)

48. Ena montana (Draparnaud, 1801)
49. Zebrina detrita (O. F. Müller, 1774)

Zebrina detrita inflata (Kobelt, 1877) \#

DAMJANOV \& LIKHAREV (1975) and DEDOV (1998) list Zebrina detrita inflata as a component of the Bulgarian fauna. The material examined by us is very variable conchologically, but differences in the genital anatomy do not justify a subspecific status. Several ecotypes of no taxonomic significance occur in Bulgaria.

50. Zebrina kindermanni kindermanni (L. Pfeiffer, 1853)

BGE

51. Zebrina varnensis (L. Pfeiffer, 1847) BLE

52. Chondrus zebra tantalus (L. Pfeiffer, 1868)

53. Multidentula ovularis (Olivier, 1801)

54. Multidentula squalina (Rossmässler, 1848)

55. Pseudochondrula seductilis (Rossmässler, 1846)

Chondrus tournefortianus (Férussac, 1821) \#

The shells found on the Bulgarian Black Sea coast in all likelihood were brought by sea currents from other regions (possibly coasts of northern Turkey).

II. Subfamily Chondrulinae

56. Chondrula (Chondrula) tridens tridens (O. F. Müller, 1774)

Chondrula (Chondrula) tridens albolimbata (L. Pfeiffer, 1859) \#

Chondrula (Chondrula) tridens bayeri (L. Pfeiffer, 1858) \# ? Besides the nominate subspecies DEDOV (1998) lists the above two subspecies which, in our opinion, do not occur in Bulgaria.

57. Chondrula (Chondrula) microtraga (Rossmässler, 1839)

In DEDOV (1998) listed as Chondrula microtraga microtraga (Rossmässler, 1839) (see also comments to species 58 and 59 below).

58. Chondrula (Chondrula) tricuspidata (Küster, 1841)

In DEDOV (1998) listed as Chondrula microtraga tricuspidata (Küster, 1841). The systematic status was changed as a result of finding several localities where the two forms live syntopically.

59. Chondrula (Chondrula) macedonica A. J. Wagner, 1915

In DEDOV (1998) listed as Chondrula macedonica macedonica.

60. Eubrephulus bicallosus (L. Pfeiffer, 1847)

61. Mastus rossmaessleri (L. Pfeiffer, 1846)

62. Mastus carneolus (Mousson, 1863)

In DEDOV (1998) listed as Mastus carneola carneola (Mousson, 1863)

63. Mastus etuberculatus (Frauenfeld, 1867) • !

In DEDOV (1998) listed as Mastus pupa (Linnaeus, 1758). Examination of material from various parts of Bulgaria showed that the species concerned was actually Mastus etuberculatus.

Mastus ponticus (Retowski, 1887) $\approx$ \#

HUDEC \& VAŠATKO (1971) found one empty shell of this species in a tributary of the Ropotamo River and 
expressed an opinion that it had been brought by sea currents from the northern coast of Turkey, and the species was not a member of Bulgarian fauna. Our view is the same.

Mastus pupa (Linnaeus, 1758) \# S

Superfamily Clausilioidea

Family Clausiliidae

Subfamily-group of the Phaedusinae

I. Subfamily Serrulininae

Tribe Serrulinini

64. Serrulina (Serrulina) serrulata (L. Pfeiffer, 1847)

65. Dobatia goettingi goettingi (Brandt, 1961)

In DEDOV (1998) listed as Dobatia goettingi (Brandt, 1961). The occurrence of the species in Bulgaria was confirmed by finding it in the Strandza Mts., far from the Black Sea coast (see also ERốsS \& FEHÉR 2001).

Subfamily-group of the Alopiinae

II. Subfamily Alopiinae

A. Tribe Medorini

66. Carinigera (Carinigera) schuetti schuetti Brandt, 1962 BLE

In DEDOV (1998) listed as Carinigera (Carinigera) schuetti Brandt, 1962.

67. Carinigera (Carinigera) buresi buresi (A. J. Wagner, 1927) BLE

67.1. Carinigera (Carinigera) buresi buresi (A. J. Wagner, 1927) ! BLE RARE

The nominate subspecies was found for the first time in the western Rhodopes and reported by IRIKOV \& MOLLOV (2006) as new to the Bulgarian fauna.

67.2. Carinigera (Carinigera) buresi damjanovi (Likharev, 1972) • BGE RARE

67.3. Carinigera (Carinigera) buresi dramaensis (Nordsieck, 1977) ! BLE RARE

This subspecies was found for the first time in the western Rhodopes and reported by IRIKOV \& MOLLOV (2006) as new to the Bulgarian fauna.

B. Tribe Cochlodinini

68. Cochlodina laminata (Montagu, 1803)

68.1. Cochlodina laminata laminata (Montagu, 1803)

68.2. Cochlodina laminata partita (Westerlund, 1892)

\section{marginata species group}

Bulgaria is especially rich in members of Macedonica, and the western Rhodopes seem to be a major centre of their origin.

69. Macedonica marginata (Rossmässler, 1835)

69.1. Macedonica marginata marginata (Rossmässler, 1835)

69.2. Macedonica marginata frivaldskyana (Rossmässler, 1839) BGE RARE

In our opinion clarifying the taxonomic status of $M$. $m$. frivaldskyana requires a detailed conchological and anatomical examination.
Macedonica marginata major (Rossmässler, 1856) \# ? Macedonica marginata balcanica (A. J. Wagner, 1927) \# ? DAMJANOV \& LIKHAREV (1975) and DEDOV (1998) list another two forms: M. marginata major (Rossmässler, 1835) and M. marginata balkanika (Rossmässler, 1835). According to IRIKOV \& MOLLOV (2006) major is a synonym of the nominate subspecies. The same probably pertains to balcanica. Nevertheless, a detailed anatomical examination of all the material of M. marginata is needed in the future, because the recently discovered conchological counterparts of the nominate form differ in the structure of genitalia.

Macedonica macedonica macedonica (Rossmässler, 1839) \# This species is known from some regions of Greece and erroneously reported by DEDOV (1998) for Bulgaria, where is does not occur.

70. Macedonica brabeneci Nordsieck, 1977 BGE

71. Macedonica hartmuti Irikov, 2003 !! BGE RARE

72. Macedonica teodorae Irikov, 2006 !! BGE RARE

\section{frauenfeldi species group}

73. Macedonica frauenfeldi (Rossmässler, 1839)

Macedonica frauenfeldi frauenfeldi (Rossmässler, 1839) \#

The nominate subspecies is listed in DEDOV (1998), but does not occur in Bulgaria.

73.1. Macedonica frauenfeldi riedeli Urbański, 1977 BGE RARE

73.2. Macedonica frauenfeldi regia Nordsieck, 1974

BGE RARE

73.3. Macedonica frauenfeldi tau Nordsieck, 1977 BLE RARE

73.4. Macedonica frauenfeldi sigma (Westerlund, 1884)

\section{- BLE RARE}

The subspecies regia, tau, and sigma are not listed in DEDOV (1998), although they occur in and were previously reported from Bulgaria.

74. Macedonica pinteri Sajó, 1968 BGE

This species is not listed in DEDOV (1998) although it was described by SAJÓ (1968) from the region of Vratca in the western Stara Planina Mts.

75. Macedonica marthae Sajó, 1968 BGE

macedonica species group

76. Macedonica pirinensis Jaeckel, 1954 • BLE

In DEDOV (1998) listed as Macedonica macedonica pirinensis Jaeckel, 1954.

77. Macedonica zilchi Urbański, 1972 BGE RARE

Subfamily group Clausiliinae

III. Subfamily Mentissoideinae

A. Tribe Filosini

78. Idyla (Idyla) castalia boschi Nordsieck, 1973 BGE Idyla (Idyla) castalia pirostoma (Boettger, 1880) \#

B. Tribe Mentissoideini

79. Euxina (Euxina) circumdata (L. Pfeiffer, 1848)

80. Euxina (Euxina) persica paulhessei (Lindholm, 1925) BGE 
81. Euxina (Euxina) pontica borisi (Hesse, 1912) • BGE 82. Galeata schwerzenbachi (L. Pfeiffer, 1848)

IV. Subfamily Clausiliinae

83. Ruthenica filograna filograna (Rossmässler, 1836)

In DEDOV (1998) listed as Ruthenica filograna (Rossmässler, 1836).

84. Micridyla pinteri (Nordsieck, 1973) BGE

Clausilia pumila C. Pfeiffer, 1828 \#

Till now there is no evidence material for the occurrence of this species in Bulgaria.

V. Subfamily Baleinae

85. Mentissella rebeli (Sturany, 1897) BGE

86. Laciniaria plicata (Draparnaud, 1801)

86.1. Laciniaria plicata plicata (Draparnaud, 1801)

86.2. Laciniaria plicata kueprijae Nordsieck, 1973 BGE RARE

86.3. Laciniaria plicata valkanovi Urbański, 1964 BGE RARE

Laciniaria plicata laticosta (Boettger, 1884) \#

Laciniaria plicata excepta (Schmidt, 1868) \# ?

Laciniaria plicata implicata (Bielz, 1851) \# ?

The last three subspecies, listed by DEDOV (1998), are not parts of the Bulgarian fauna.

87. Laciniaria macilenta (Rossmässler, 1842) BGE

88. Laciniaria bajula (Schmidt, 1968) BGE

88.1. Laciniaria bajula bajula (Schmidt, 1968) BGE

88.2. Laciniaria bajula lunella Nordsieck, 1973 BGE

88.3. Laciniaria bajula mursalicae (Urbański, 1969)

BGE RARE

88.4. Laciniaria bajula ditrichi Nordsieck, 1973 口 BGE

RARE

Subspecies bajula and lunella are not listed in DEDOV (1998), although URBAŃSKI (1969) and NORDSIECK (1973) describe them from the regions of the Rhodopes and Rila Mts., respectively.

89. Alinda (Alinda) biplicata (Montagu, 1803)

89.1. Alinda (Alinda) biplicata biplicata (Montagu, 1803)

89.2. Alinda (Alinda) biplicata michaudiana (L. Pfeiffer, 1948) ○ BLE

Alinda biplicata eupleuris (Moellendorff, 1899) \# Alinda biplicata euptychia (Ehrmann, 1960) \# Alinda biplicata distinctior (A. J. Wagner, 1915) \# S

According to NORDSIECK (personal communication) the specimens from Bulgaria recorded as $A$. $b$. distinctior actually represent Alinda biplicata michaudiana.

90. Alinda (Alinda) atanasovi (Urbański, 1964)

This species was described for the second time by URBAŃSKI (1969) as Laciniaria biplicata tenuispira and on another occasion by DEDOV \& NEUBERT (2006) as Laciniaria potochensis. According to NORDSIECK (2008) both these names are synonyms of Alinda atanasovi.

91. Alinda (Alinda) vratzatica (Likharev, 1972) • BGE
92. Alinda (Alinda) wagneri (A.J. Wagner, 1911) • BGE 92.1. Alinda (Alinda) wagneri wagneri (A. J. Wagner, 1911) - BGE RARE

92.2. Alinda (Alinda) wagneri petrohanica Urbański, 1969 • BGE RARE

93. Alinda (Pseudalinda) serbica serbica (Moellendorf, 1873) • BLE

94. Balea kaeufeli (Brandt, 1962) BGE RARE

95. Balea eninskoensis Irikov, 2006 !! BGE RARE

Balea perversa (Linnaeus, 1758) \#

This species is not easy to distinguish from other Balea species in Bulgaria and in all likelihood all of its records refer to Balea kaeufeli.

96. Vestia (Brabenenecia) ranojevici ranojevici (Pavlovic, 1912) BGE

Vestia riloensis (A. J. Wagner, 1915) S

97.1. Vestia (Vestiella) roschitzi trigonostoma (Pavlovic, 1912) BLE

97.2. Vestia (Vestiella) roschitzi nordsieckiana (Urbański, 1979) BLE

Vestia petrovici (Pavlovic, 1912) \# ?

98. Bulgarica (Strigilecula) vetusta vetusta (Rossmässler, 1836) RARE

DEDOV (1998) listed it as Bulgarica (Strigilecula) vetusta.

99. Bulgarica (Bulgarica) varnensis (L. Pfeiffer, 1848) BLE

99.1. Bulgarica (Bulgarica) varnensis varnensis (L. Pfeiffer, 1848) BLE

99.2. Bulgarica (Bulgarica) varnensis gabrovnitsana Irikov, 2006 !! BGE RARE

99.3. Bulgarica (Bulgarica) varnensis trimontsiana Irikov, 2006 !! BGE RARE

100. Bulgarica (Bulgarica) fritillaria (Frivaldsky, 1835)

BGE

101. Bulgarica (Bulgarica) fraudigera (Rossmässler, 1839) BGE

102. Bulgarica (Bulgarica) pseudofraudigera Nordsieck, 1973 • BGE RARE

103. Bulgarica (Bulgarica) hiltrudae Nordsieck, 1974

BGE

104. Bulgarica (Bulgarica) urbanskii Nordsieck, 1973

BGE

104.1. Bulgarica (Bulgarica) urbanskii urbanskii Nordsieck, 1973 • BGE

104.2. Bulgarica (Bulgarica) urbanskii paganella Nordsieck, 1974 BGE

105. Bulgarica (Bulgarica) bulgariensis (L. Pfeiffer, 1848) - BGE

105.1. Bulgarica (Bulgarica) bulgariensis bulgariensis (L. Pfeiffer, 1848) • BGE RARE

105.2. Bulgarica (Bulgarica) bulgariensis intricata (Mousson, 1859) - BGE RARE

105.3. Bulgarica (Bulgarica) bulgariensis osmanica (Westerlund, 1884) • BGE RARE

106. Bulgarica (Bulgarica) denticulata thessalonica (Rossmässler, 1839) • BLE 
Bulgarica (Bulgarica) rugicollis (Rossmässler, 1836) \# This species occurs in Romania but in Bulgaria it is probably confused with $B$. pseudofraudigera.

Superfamily Succineioidea

Family Succineidae

107. Succinea putris (Linnaeus. 1758) RARE

108. Succinea oblonga Draparnaud, 1801

109. Oxyloma elegans (Risso, 1826)

110. Oxyloma sarsii (Esmark et Hoyrr, 1886) !

111. Oxyloma dunkeri (L. Pfeiffer, 1865) !

The last two species are recorded by GEORGIEV (in press) as new to the Bulgarian fauna.

Superfamily Achatinoidea

Family Ferussaciidae

112. Cecilioides acicula (O. F. Müller, 1774)

113. Cecilioides jani (De Betta et Martinati, 1855)

114. Cecilioides spelaeus (A. J. Wagner, 1914) BLE

Family Subulinidae

115. Rumina decollata (Linnaeus, 1758) RARE

Superfamily Testacelloidea \#

Family Oleacinidae \#

Poiretia algira (Bruguiere, 1972) \#

Till now there is no unambiguous evidence material for the presence of this species in Bulgaria.

Superfamily Punctacea

Family Punctidae

I. Subfamily Punctinae

116. Punctum pygmaeum (Draparnaud, 1801)

II. Subfamily Goniodiscinae

117. Discus ruderatus (Férussac, 1821) RARE

118. Discus perspectivus (Megerle von Mühlfeld, 1816)

RARE

Family Gastrodontidae

119. Zonitoides nitidus (O. F. Müller, 1774)

Superfamily Helicarionoidea

Family Euconulidae

120. Euconulus fulvus (O. F. Müller, 1774)

Superfamily Vitrinoidea

Family Vitrinidae

121. Vitrina pellucida (O. F. Müller, 1774)

122. Eucobresia diaphana (Draparnaud, 1805)

123. Gallandia annularis (Studer, 1820)

124. Oligolimax reitteri (Boettger, 1880) • BGE RARE

Family Zonitidae

I. Subfamily Vitreininae

125. Vitrea diaphana (Studer, 1829)

In DEDOV (1998) listed as Vitrea diaphana diaphana. 126. Vitrea transsylvanica (Clessin, 1877) RARE
127. Vitrea subrimata (Reinhardt, 1871)

128. Vitrea neglecta Damjanov et L. Pintér 1969

Vitrea bulgarica Damjanov et L. Pintér 1969 S \#

The species is synonymised (Vitrea bulgarica is a synonym of Vitrea neglecta) as a result of detailed anatomical investigations of the genital system (IRIKOV 2001). Nevertheless some authors (DEDOV 2008) still use the name as valid without refutation of the synonymisation or another justification.

129. Vitrea contracta (Westerlund, 1871)

130. Vitrea riedeli Damjanov et L. Pintér 1969

131. Vitrea pygmaea (Boettger, 1880)

132. Vitrea vereae Irikov, Georgiev et Riedel, 2004 BGE

RARE

Vitrea sturanyi (A. J. Wagner, 1907) \#

This species is included in DEDOV (1998) although RIEDEL (1975, 1980, 1998) and PINTÉR (1972) point out that it does not occur in Bulgaria.

133. Spinophallus uminskii Riedel, 1960 • BGE RARE

II. Subfamily Zonitinae

134. Aegopinella pura (Alder, 1830)

135. Aegopinella minor (Stabile, 1864)

136. Aegopinella nitens (Michaud, 1831) I

137. Oxychilus (Oxychilus) draparnaldi (Beck, 1837) I

138. Oxychilus (Ortizius) translucidus (Mortilet, 1854)

139 Oxychilus (Mediterranea) hydatinus (Rossmässler, 1838)

140. Oxychilus (Longiphallus) deilus rumelicus (Hesse, 1913)

141. Oxychilus (Spinophallus) investigatus Riedel, 1993

142. Oxychilus (Morlina) glaber striarius (Westerlund, 1881)

143. Oxychilus (Morlina) urbanskii Riedel, 1963

144. Oxychilus (Riedelius) inopinatus (Uličny, 1887)

145. Oxychilus (Riedelius) depressus (Sterki, 1880)

Oxychilus moussoni (Kobelt, 1878) S \#

This species is listed in DEDOV (1998) as Oxychilus moussoni though the material from Bulgaria examined by RIEDEL \& MAASSEN (1993) was classified as representing $O$. investigatus.

Oxychilus camelinus tracicus (Hesse, 1913) \#

146. Balcanodiscus frivaldskyanus (Rossmässler, 1848)

- BLE

147. Nesovitrea hammonis (Ström, 1765)

III. Subfamily Daudebardiinae

148. Daudebardia (Daudebardia) brevipes (Draparnaud, 1805)

149. Daudebardia (Daudebardia) rufa cycladum Martens, 1889

150. Daudebardia (Libania) wiktori Riedel, 1967

151. Carpathica (Illyrica) bielawskii Riedel, 1963 BLE

152. Carpatica (Illyrica) stussineri (A. J. Wagner, 1895)

153. Carpatica ?wirthi (Forcart, 1971) ! ?

This species was recorded from the Rhodopes only based on shells, by IRIKOV \& MOLLOV (2006), but the 
authors realise the necessity for anatomical examination in order to clarify its taxonomic status.

Superfamily Arionoidea

Family Arionidae

154. Arion lusitanicus Mabille, 1868 I

155. Arion subfuscus (Draparnaud, 1805)

156. Arion silvaticus Lohmander, 1937

157. Arion hortensis Férussac, 1819 I

158. Arion fasciatus (Nilsson, 1822) I

Family Milacidae

159. Milax parvulus Wiktor, 1968 BGE

160. Milax verrucosus Wiktor, 1969 BGE

161. Tandonia kusceri (H. Wagner, 1931) BLE

162. Tandonia serbica (H. Wagner, 1931) BLE

163. Tandonia budapestensis (Hazay, 1881)

164. Tandonia piriniana Wiktor, 1983 BGE RARE

165. Tandonia cristata (Kaleniczenko, 1851)

166. Tandonia pinteri (Wiktor, 1975) BGE RARE

167. Tandonia totevi (Wiktor, 1975) BGE RARE

Tandonia rustica (Millet, 1843) \#

Superfamily Limacoidea

Family Limacidae

168. Limax (Limax) maximus Linnaeus, 1758

169. Limax (Limax) subalpinus Lessona, 1880 RARE

This species, found in two localities in the Western

Rhodopes (IRIKOV \& MOLLOV 2006), has a very characteristic genital structure.

170. Limax (Limax) cinereoniger Wolf, 1803

171. Limax (Limax) punctulatus Sordelli, 1870

172. Limax (Limax) macedonicus Hesse, 1928 BLE

173. Limax (Limax) conemenosi Boettger, 1882 BLE

174. Limax (Limacus) flavus Linnaeus, 1758

175. Limax (Limacus) maculatus (Kaleniczenko, 1851)

In DEDOV (1998) the species is listed as Limax (Limacus) ecarinatus (Kaleniczenko, 1851).

176. Lehmania nyctelia (Bourguignat, 1861)

177. Lehmania brunneri (H. Wagner, 1931) BLE

178. Lehmania horezia Grosu et Lupu, 1962 RARE

Lehmania sarmizegetusae Grossu, 1970 S \#

This species is a synonym of Lehmania horezia.

179. Malacolimax tenellus (O. F. Müller, 1774) RARE

Family Agriolimacidae

180. Deroceras (Deroceras) laeve (O. F. Müller, 1774)

181. Deroceras (Deroceras) sturanyi (Simroth, 1894)

182. Deroceras (Deroceras) panormitanum (Lessona et Pollonera, 1882)

183. Deroceras (Agriolimax) turcicum (Simroth, 1894)

184. Deroceras (Agriolimax) reticulatum (O. F. Müller, 1774)

185. Deroceras (Agriolimax) thersites (Simroth, 1886)

BLE RARE

186. Deroceras (Agriolimax) agreste (Linnaeus, 1758)
Deroceras agreste transcaucasicum Hudec et Vašatko, 1971 \# ?

The species was listed in DEDOV (1998) with the nominate subspecies Deroceras agreste agreste and another subspecies, Deroceras agreste trancaucasicum, whose systematic status is unclear.

187. Deroceras (Agriolimax) pageti Grossu, 1972

188. Deroceras (Plathystimulus) zilchi Grossu, 1969 BLE RARE

This species was found in two localities in the eastern Stara Planina Mts (village Neikovo, 18.05.2002, leg. Ê. ÊIROV) and is easily distinguishable due to its anatomical characters.

189. Deroceras (Plathystimulus) bulgaricum Grossu, 1969 BGE RARE

190. Deroceras (Liolytopelte) bureschi (H. Wagner, 1934) BLE

191. Krynickillus urbanskii (Wiktor, 1971) BLE

Superfamily group Helicoidea

Superfamily Hygromioidea

Family Helicodontidae

I. Subfamily Lindholmiolinae

192. Lindholmiola girva (Frivaldsky, 1835) BLE

This species is listed in DEDOV (1998) as the nominate subspecies Lindholmiola girva girva (see the next species).

193. Lindholmiola pirinensis Jaeckel, 1954 BLE

This species is listed in DEDOV (1998) as Lindholmiola pirinensis pirinensis. The two species were earlier regarded as subspecies, but they are sympatric in the western Rhodopes and thus are most likely to be distinct species.

II. Subfamily Helicodontinae

194. Soosia diodonta (Férussac, 1821) RARE

GEORGIEV \& STOYCHEVA (2007) provide a new Bulgarian record of this rare species.

Family Hygromiidae

I. Subfamily Trichiinae

Tribe Trichiini

195. Trichia hispida (Linnaeus, 1758) RARE

196. Trichia erjaveci (Brusina, 1870)

Tribe Helicellini

197. Xerolenta obvia (Menke, 1828)

Xerolenta obvia razlogi (L. Pintér, 1969) ? S \#

Xerolenta obvia dobrudschae (Kobelt, 1877) ? S \#

Xerolenta obvia depulsa (L. Pintér, 1969) ? S \#

Xerolenta obvia pappi (Schütt, 1962) ? S \#

DEDOV (1998) listed X. obvia razlogi and X. obvia dobrudschae, but in our opinion they are only variations of the very polymorphic nominate subspecies. The same pertains to X. obvia depulsa and X. obvia pappi.

198. Xerolenta macedonica Hesse, 1928 • BLE

199. Xerolenta spiruloides (A. J. Wagner, 1916) • BGE 
200. Helicopsis striata (O. F. Müller, 1774)

201. Helicopsis dejecta (Jan, 1832) RARE

202. Helicopsis instabilis (Rossmässler, 1838) RARE

203. Candidula rhabdotoides (A. J. Wagner, 1927) BLE

204. Xeropicta krynickii (Krynicki, 1833)

205. Xeropicta derbentina (Krynicki, 1836)

II. Subfamily Hygromiinae

206. Pseudotrichia rubiginosa (Schmidt, 1853)

207. Perforatella incarnata (O. F. Müller, 1774)

208. Cernuella virgata variabilis (Draparnaud, 1801)

209. Cernuella cisalpina (Rossmässler, 1837)

III. Subfamily Euomphaliini

210. Monacha (Monacha) claustralis (Menke, 1828) !

211. Monacha (Monacha) cartusiana (O. F. Müller, 1774)

212. Monacha (Monacha) carascaloides (Bourguignat, 1855)

213. Monacha (Monacha) solidior (Mousson, 1873) !

214. Monacha (Monacha) ovularis (Bourguignat, 1855)

- !

215. Monacha (Monacha) venusta (L. Pintér, 1968)

216. Monacha (Monacha) ocellata (L. Pintér, 1968) !

217. Monacha (Monacha) oshanovae I. Pintér et L. Pintér, 1970 ?

According to IRIKOV (2008) the taxonomic separation of this species is very uncertain and here it is only provisionally listed as a member of the Bulgarian fauna.

218. Euomphalia strigella (Draparnaud, 1801)

Euomphalia strigella mehadiae (Bourguignat, 1881) \# Besides the nominate subspecies, DEDOV (1998) lists also Euomphalia strigella mehadiae, in our opinion the distinction between the two forms is doubtful.

Family Cochlicellidae

219. Cochlicella acuta (O. F. Müller, 1774) I

Superfamily Xanthonychoidea

Family Bradybaenidae

\section{REFERENCES}

Claus E. 1977. Beitrag zur Molluskenfauna der Rhodopen Bulgariens. Malak. Abh. 5: 307-312.

DAmJANOV S., LikHAREV I. 1975. Fauna Bulgarica, Gastropoda terrestria. IV. Bulgarian Academy of Sciences, Sofia.

DEDOV I. 1998. Anotated check-list of the Bulgarian terrestrial snails (Mollusca, Gastropoda). Linzer biol. Beitr. 30: $745-765$.

Dedov I. 2007. On the status of the Bulgarian Pyramidula (Gastropoda: Pulmonata: Pyramidulidae). Acta Zool. Bulgar. 59: 221-224.

DEDOV I. 2008. Terrestrial gastropods (Mollusca, Gastropoda) of the Bulgarian part of the Alibotush Mts. Malac. Bohemoslov. 7: 17-20.

\section{Bradybaena fruticum (O. F. Müller, 1774)}

Superfamily Helicoidea

Family Helicidae

I. Subfamily Ariantinae

221. Arianta arbustorum (Linnaeus, 1758) RARE

222. Arianta pelia (Hesse, 1912) BGE

223. Cattania trizona (Rossmässler, 1835)

224. Cattania balcanica (Kobelt, 1876) • BLE

225. Cattania rumelica (Rossmässler, 1835) • BLE

226. Cattania haberhaueri (Sturany, 1897) • BGE

227. Cattania kattingeri (Knipper, 1941) B BLE RARE

228. Cattania ardica Dedov et Subai, 2006 - BGE ! !

229. Wladislawia polinskii (A. J. Wagner, 1927) BGE

230. Wladislawia sztolcmani (A. J. Wagner, 1927) BGE

II. Subfamily Helicinae

231. Helix (Helix) lucorum Linnaeus, 1758

Helix lucorum onixiomitra Bourguignat, 1860 S \#

Helix lucorum castanea Olivier, 1801 S \#

DEDOV (1998) lists these two subspecies, which in our opinion are synonyms of the nominate form.

232. Helix (Helix) pomatia Linnaeus, 1758

Helix pomatia rhodopensis Kobelt, 1906 S \#

Helix pomatia serbica Kobelt, 1906 S \#

The two subspecies, recorded by DEDOV (1998), are in our opinion synonyms of the nominate form.

233. Helix (Helix) albescens Rossmässler, 1839

Helix vulgaris Rossmässler, 1839 S \#

See comment on the next species.

234. Helix (Helix) philibinensis Rossmässler, 1839

Helix vulgaris Rossmässler, 1839 S \#

The last two species - albescens and philibinensis - till now in Bulgaria were considered collectively under the name Helix vulgaris.

235. Helix (Pelasga) pomacella Mousson, 1854

236. Helix (Pelasga) figulina Rossmässler, 1839

237. Helix (Cornu) aspersa (O. F. Müller, 1774) RARE

238. Eobania vermiculata (O. F. Müller, 1774)

239. Cepaea vindobonensis (Férussac, 1821)

DEDOV I., NeUBERT E. 2006. Description of a new species of Clausiliidae from the Eastern Rhodopes Mountains, Bulgaria (Mollusca: Gastropoda: Clausiliidae). Arch. Moll.. 135: 73-79.

ERÖSS Z., FEHÉR Z. 2001. Dobatia goettingi Brandt, 1961 (Gastropoda: Clausiliidae) in Bulgaria. Opusc. Zool. 33: 139-142.

GEORGIEV D. in press. Two new species from the family Succineidae (Beck, 1837) (Gastropoda: Pulmonata) for the Bulgarian fauna. Trav. Sci. Univ. Plovdiv. Animalia 42.

Georgiev D., Stoycheva S. 2007. A new record of Soosia diodonta (Férussac, 1821) (Gastropoda: Pulmonata: 
Helicodontidae) in Bulgaria. Malac. Bohemoslov. 6: 35-37.

GitTenberger E., BANK R. 1996. A new start in Pyramidula (Gastropoda: Pulmonata: Pyramidulidae). Basteria 60: 71-78.

HudEC V., VAŠATKO J. 1971. Beitrag zur Molluskenfauna Bulgariens. Acta Sc. Nat. Brno 5: 1-38.

HudEC V., VAŠATKO J. 1973. Zur Kenntnis Molluskenfauna Bulgariens. Acta Sc. Nat. Brno 7: 1-33.

IRIKOV A. 2001. To the knowledge of the anatomy and taxonomy of two species from genus Vitrea Fitzinger, 1833 (Gastropoda, Zonitidae). Trav. Sci. Univ. Plovdiv. Animalia 37: 35-38.

IRIKOV A. 2003. A new species of Macedonica O. Boettger, 1877 from Bulgaria (Gastropoda: Pulmonata: Clausiliidae). Arch. Moll. 132: 143-145.

IRIKOV A. 2006. New taxa of Clausiliidae from Bulgaria (Gastropoda: Pulmonata: Clausiliidae). Arch. Moll. 135: 81-89.

IRIKOV A. in press [2008]. Truncatellina rothi (Reinhardt, 1916) (Gastropoda: Vertiginidae: Truncatellininae) - a new species for the Bulgarian malacofauna. Acta Zool. Bulgar.

IRIKOV A., GEORGIEV D. in press [2008]. A review of the Agardhiella species in Bulgaria (Gastropoda: Pulmonata: Argnidae). Acta Zool. Bulgar.

IRIKOV A., GEORGIEV D., RIEDEL A. 2004. A new species of the genus Vitrea Fitzinger, 1833 from Bulgaria (Gastropoda: Pulmonata : Zonitidae). Folia Malacol. 12: 79-81.

IRIKOV A., MOLlov I. 2006. Terrestrial gastropods (Mollusca: Gastropoda) of the Western Rhodopes (Bulgaria). In: Biodiversity of Bulgaria (BERON P., ed). vol. 3. Biodiversity of Western Rhodopes (Bulgaria and Greece) I. Pensoft et Nat. Mus. Natur. Hist., Sofia, pp. 753-832.

JACKIEWICZ M. 1979. Materials to Aciculidae fauna of Balkan peninsula (Mollusca, Prosobranchia). Bull. Soc. Amis Sci. Lettr. Poznań, D 18 [1978]: 135-138.

KÖRNIG G. 1983. Beitrag zur Ökologie und Zoogeographie bulgarischer Landgastropoden. Malak. Abh. 9: 31-52.

NoRDSIECK H. 1970. Die Chondrina-Arten der dinarischen Länder. Arch. Moll. 100: 243-261.
NORDSIECK H. 1973. Neue Balkanformen der Mentissoideinae und Baleinae. Zur Anatomie und Systematik der Clausilien. XIII. Arch. Moll. 103: 179-208.

NORDSIECK H. 1974. Neue Clausilien der Balkanhalbinsel. Zur Anatomie und Systematik der Clausilien. XV. Arch. Moll. 104: 123-170.

NORDSIECK H. 1977. Zur Anatomie und Systematik der Clausilien. XVIII. Neue Taxa rezenter Clausilien. Arch. Moll. 108: 73-107.

NORDSIECK H. 2008. Alinda biplicata and Laciniaria plicata, diversity in comparison. http://www.clausilia.de

PINTÉR L. 1972. Die Gattung Vitrea Fitzinger, 1833 in den Balkanländer (Gastropoda: Zonitidae). Ann. Zool. 29: 209-315.

RIEDEL A. 1975. Die Zonitiden-Fauna Bulgariens (Gastropoda), ihre Herkunft und Verbreitung. Fragm. Faun. 20: 157-177.

RIEDEL A. 1980. Eine weitere Zonitiden-Art (Gastropoda) mit vaginalen Anhangsorgan. Ann. Zool. 35: 486-495.

RIEDEL A. 1998. Genera Zonitidarum - Addenda et corrigenda. (Gastropoda, Stylommatophora). Museum and Institute of Zoology, Polish Academy of Sciences, Warsaw.

Riedel A., MAassen M. J. W. 1993. Berichtigung zur Frage von Hyalina moussoni Kobelt, 1878 (Gastropoda, Pulmonata, Zonitidae). Basteria 57: 139-146.

SAJó I. 1968. Zur Clausiliidenfauna Bulgariens (Gastropoda). Acta Zool. Acad. Sci. Hungar. 14: 447-454.

URBAŃSKI J. 1960. Neue Landschnecken aus Bulgarien (Orculidae u. Pupiliidae, Moll. Pulm.). (Systematische, zoogeographische und ökologische Studien über die Mollusken der Balkan-Halbinsel. IV). Bull. Soc. Amis Sci. Lettr. Poznań, D 1: 57-67.

URBAŃSKI J. 1969. Bemerkenswerte Balkanische Stylommatophoren (Systematische, Zoogeographische und Ökologische Studien über die Mollusken der Balkan-Halbinsel. IX. Bull. Soc. Amis Sci. Lettr. Poznań, D 9: 225-262.

Received: , 2008

Accepted: December 15th, 2008 